\title{
Biochemical Diagnosis and Localization of Pheochromocytoma
}

\section{Can We Reach a Consensus?}

ASHLEY GROSSMAN,${ }^{a}$ KAREL PACAK,${ }^{b}$ ANNA SAWKA,${ }^{c}$ JACQUES W. M. LENDERS, ${ }^{d}$ DEBRA HARLANDER, ${ }^{e}$ ROBERT T. PEASTON,${ }^{f}$ RODNEY

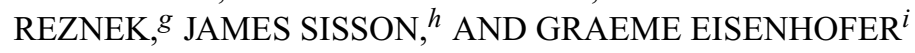

${ }^{a}$ Department of Endocrinology, St. Bartholomew's Hospital, West Smithfield, London, UK

${ }^{b}$ Reproductive Biology and Medicine Branch, National Institute of Child Health and Human Development, National Institutes of Health, Bethesda,

Maryland 20892, USA

${ }^{c}$ Department of Medicine, University of Toronto, Toronto, Ontario, Canada

${ }^{d}$ Department of General Internal Medicine, St. Radboud University Hospital, Nijmegen, the Netherlands

${ }^{e}$ Pheochromocytoma Support Group, Camden, New York 13316, USA

$f^{f}$ Department of Clinical Biochemistry, Freeman Hospital, Newcastle upon Tyne, $U K$

${ }^{g}$ Division of Radiology, St. Bartholomew's Hospital, West Smithfield, London, UK

${ }^{h}$ Department of Radiology, University of Michigan Hospitals, Ann Arbor, Michigan 48109, USA

${ }^{i}$ Clinical Neurocardiology Section, National Institute of Neurological Disorders and Stroke, National Institutes of Health, Bethesda, Maryland 20892, USA

Abstract: Pheochromocytomas can have a highly variable presentation, making diagnosis challenging. To think of the tumor represents the crucial initial step, but establishing the diagnosis requires biochemical evidence of excessive catecholamine production and imaging studies to localize the source. Currently, however, there exist no generally agreed upon guidelines based on which tests and testing algorithms should be used to confirm and locate or exclude a suspected pheochromocytoma. Choice of biochemical tests and imaging studies instead usually depends on institutional experience. At the First International Symposium on Pheochromocytoma (ISP2005), held in Bethesda in October 2005, a panel of experts and patient representatives discussed current problems and available options for tumor diagnosis and localization and formulated

Address for correspondence: Ashley Grossman, M.D., F.R.C.P., FMed. Sci., Department of Endocrinology, St. Bartholomew's Hospital, West Smithfield, London, EC1A 7BE, UK. Voice: 4420760 18343, fax: 442076018505.

e-mail: a.b.grossman@qmul.ac.uk

Ann. N.Y. Acad. Sci. 1073: 332-347 (2006). (c) 2006 New York Academy of Sciences. doi: 10.1196/annals.1353.038 
recommendations, which were subsequently agreed upon by those in attendance at the meeting. This article summarizes the discussion and recommendations derived from that session.

KEYWORDS: pheochromocytoma; biochemical diagnosis; metanephrines; catecholamines; localization; computed tomography; magnetic resonance imaging; MIBG; positron emission tomography

\section{INTRODUCTION}

Pheochromocytomas, although a rare cause of hypertension, are dangerous but usually surgically curable tumors that require consideration among large numbers of patients, at considerable cost to healthcare systems. Among overall numbers of patients tested for pheochromocytoma, less than $1 \%$ actually harbor the tumor, but, based on autopsy studies, probably as many cases or more are missed than are diagnosed. ${ }^{1-3}$ Thus, although frequently searched for and rarely found, the tumor remains an overlooked clinical entity.

As discussed by Dr. William Manger (New York University Medical Center, New York) in the keynote address at the First International Symposium on Pheochromocytoma (ISP2005), diagnosis of the tumor first requires recognition of the varied clinical conditions and situations where pheochromocytoma should be considered. To consider the possibility of the tumor represents the crucial initial step, but confirming the diagnosis requires biochemical measurements of catecholamines and catecholamine metabolites in urine or plasma, followed by imaging studies to localize the source preparatory to surgical removal.

A major problem in reaching or excluding the diagnosis of pheochromocytoma is that there are no generally agreed upon guidelines about how and which biochemical tests and imaging studies should best be carried out, interpreted, and followed-up. Practices vary among different centers, and recommendations are more often based on institutional experience than on evidence-based medicine. Representatives of patient support groups at ISP2005 also indicated that there was a need for improved education of clinicians in how to recognize, diagnose, and localize the tumor, and they expressed frustration that progress seemed hampered by lack of agreement and conflicting views from experts in the field.

A "breakout session" was therefore held during ISP2005 with the aim of fostering resolution of issues and problems associated with biochemical diagnosis and localization of pheochromocytoma. More than 40 participants, including leading experts in the field and representatives from patient support groups, attended the session. Under the guidance of impartial moderators (Dr. Ashley Grossman, St Bartholomew's Hospital, London, UK, and Dr. Hendrik Lehnert, University of Warwick, UK), participants were asked to formulate recommendations that were subsequently discussed and agreed upon at a 
final general session of this symposium where all attendees could be present. Recommendations were based on answers to a series of questions, as detailed below.

- What are the preferred biochemical tests or testing algorithms for confirming or excluding pheochromocytoma and what cost-benefit factors should be considered?

- What precautions should be considered to minimize or avoid falsepositive results or drug interference during biochemical testing and imaging procedures?

- What evidence for the presence of a tumor justifies imaging studies?

- What imaging strategies are appropriate for localization of pheochromocytoma and in what order should they be applied?

This report reviews the discussion held at the breakout session, where possible outlines generally agreed upon recommendations, and where not possible seeks to account for why there may be no clear answer and what future advances may assist in reaching an answer. Because the presentation of pheochromocytoma can be variable and complex, ${ }^{4}$ which exerts a subsequent impact on the choice of biochemical tests and imaging strategies, outlined recommendations are not intended as strict guidelines, but as complements to sound clinical judgment.

\section{BIOCHEMICAL DIAGNOSIS}

An important recommendation agreed upon by all attending participants at the breakout and final discussion sessions of ISP2005 was that initial biochemical testing for pheochromocytoma should include measurements of plasma concentrations or urinary excretion of fractionated metanephrines (separate measurements of normetanephrine and metanephrine) wherever these tests are available. Importantly, this recommendation does not exclude the additional use of other tests (e.g., urinary or plasma catecholamines) or measurements of both plasma and urinary fractionated metanephrines during initial testing for the tumor. Whereas other tests are used during initial testing, these should be used in conjunction with measurements of fractionated metanephrines in plasma or urine.

The importance of measurements of urinary or plasma fractionated metanephrines for the diagnosis of pheochromocytoma is principally based on research by National Institutes of Health (NIH) investigators establishing that pheochromocytomas contain catechol- $O$-methyltransferase, the presence of which results in the metabolism of catecholamines to metanephrines within tumor cells. ${ }^{5-7}$ It is further proposed that production of metanephrines within tumor cells is continuous and more accurately reflects tumor mass than release of catecholamines, which can occur episodically or not at all in nonsecretory tumors. Consistent with these concepts, it has now been established by four independent groups of investigators that measurements of plasma 
TABLE 1. Sensitivity and specificity of measurements of plasma fractionated metanephrines for diagnosis of pheochromocytoma

\begin{tabular}{|c|c|c|c|c|c|c|}
\hline \multirow[b]{2}{*}{ Center } & \multicolumn{2}{|c|}{ Study Cohort $(n)$} & \multicolumn{2}{|c|}{ URL (nmol/L) } & \multicolumn{2}{|c|}{$\begin{array}{c}\text { Diagnostic } \\
\text { Performance }\end{array}$} \\
\hline & Pheo & No pheo & NMN & $\mathrm{MN}$ & Sensitivity & Specificity \\
\hline Vienna $^{9}$ & 17 & 14 & 0.66 & 0.31 & $100 \%$ & $100 \%$ \\
\hline $\mathrm{NIH}^{10}$ & 214 & 644 & $\begin{array}{l}0.61 \\
2.19\end{array}$ & $\begin{array}{l}0.31 \\
1.20\end{array}$ & $\begin{array}{l}99 \% \\
79 \%{ }^{a}\end{array}$ & $\begin{array}{r}89 \% \\
100 \%\end{array}$ \\
\hline Mayo Clinic ${ }^{12}$ & 56 & 445 & 0.90 & 0.50 & $96 \%$ & $85 \%$ \\
\hline University of Essen ${ }^{13}$ & 24 & 126 & 0.69 & 0.19 & $96 \%$ & $80 \%$ \\
\hline Freeman Hospital $^{b}$ & 11 & 114 & 0.90 & 0.55 & $100 \%$ & $91 \%$ \\
\hline Cleveland Clinic $^{c}$ & 32 & 0 & $\begin{array}{l}0.90 \\
2.19\end{array}$ & $\begin{array}{l}0.50 \\
1.20\end{array}$ & $\begin{array}{l}97 \% \\
84 \% a\end{array}$ & - \\
\hline All centers & 354 & 1,343 & & & $98 \%$ & $87 \%$ \\
\hline
\end{tabular}

$\mathrm{URL}=$ upper reference limits of reference intervals; $\mathrm{NMN}=$ normetanephrine; $\mathrm{MN}=$ metanephrine.

${ }^{a}$ Denotes values of diagnostic sensitivity calculated using upper reference limits adjusted to provide $100 \%$ specificity; indicates percentage of patients where there is close to $100 \%$ probability of a pheochromocytoma.

${ }^{b}$ Peaston, R.T., Neely, R.D.G. 2005. Biochemical diagnosis of pheochromocytoma: the role of plasma metanephrines. Proceedings of the First International Symposium on Pheochromocytoma [abstract]. 150.

${ }^{c}$ Bravo, E.L., Bravo, T. 2005. Clinical characteristics of incidentally discovered pheochromocytoma from an analysis of 50 proven cases. Proceedings of the First International Symposium on Pheochromocytoma [abstract] 27. This volume.

metanephrines provides superior diagnostic sensitivity over measurements of plasma or urinary catecholamines for the detection of pheochromocytoma. ${ }^{8-13}$ A further study presented at ISP2005 by Dr. Robert Peaston (Freeman Hospital, Newcastle upon Tyne, UK) provided documentation by a fifth group that measurements of plasma metanephrines provide improved accuracy over urinary catecholamines for the diagnosis of the tumor (TABLE 1).

Several studies involving measurements of urinary fractionated metanephrines have similarly indicated that these tests provide superior diagnostic sensitivity over urinary or plasma catecholamines, urinary vanillylmandelic acid, or total metanephrines, the latter measured as the combined sum of normetanephrine and metanephrine by early spectrophotometric methods. ${ }^{10,14-17}$ Taken together, the weight of accumulating evidence clearly indicates that measurements of fractionated metanephrines in urine or plasma provide superior diagnostic sensitivity over urinary or plasma measurements of catecholamines and other catecholamine metabolites.

There was no consensus at the meeting as to whether measurements of plasma or urinary fractionated metanephrines offer the preferred test, although evidence by two different groups has indicated that the plasma test may offer diagnostic advantages over the urine test. ${ }^{10,13}$ Against this, Dr. Emmanuel Bravo (Cleveland Clinic, $\mathrm{OH}$ ) presented evidence at ISP2005 that measurements of urinary fractionated metanephrines were superior to plasma-free 
TABLE 2. Relative merits and demerits of measurements of urinary fractionated and plasma-free metanephrines for the diagnosis of pheochromocytoma

\begin{tabular}{ll}
\hline Urinary Fractionated Metanephrines & Plasma-Free Metanephrines \\
\hline Well-established, widely available test & Relatively new test with limited availability \\
Urinary concentrations (200-2,000 nmol) & Plasma concentrations (0.1-0.5 nmol) can \\
make analysis relatively easy & make analysis difficult \\
Easy for clinicians to implement with & Blood collections require some time and \\
minimal expenditure of time and effort & effort by medical staff \\
Twenty-four-hour collections can be & Blood sampling relatively more convenient \\
inconvenient for patients & for patients \\
Problems with reliability of incomplete & Collection and handling of blood samples \\
timed urine collections & can be better regulated \\
Difficult to control dietary and daily life & Influences of diet and sympathoadrenal \\
influences on sympathoadrenal function & function more easily controlled \\
In children 24-h collections are difficult & In children blood sampling may be \\
and results are difficult to interpret & stressful, but results are more easily \\
without age-appropriate reference & interpreted without age-appropriate \\
intervals & reference intervals \\
Urine collections may be inappropriate in & Test is applicable in patients with renal \\
patients with renal failure & failure \\
\hline
\end{tabular}

metanephrines for the diagnosis of incidentally discovered pheochromocytomas; the urinary test returned positive results in $96 \%$ of patients with the tumor compared to $84 \%$ for the plasma test. The latter value, however, was obtained using upper limits of reference intervals identical to those described elsewhere that had been adjusted to provide $100 \%$ specificity. ${ }^{10}$ Thus, the results of the study by Bravo may be reinterpreted to indicate that $84 \%$ of the patients with pheochromocytoma had elevations of plasma metanephrines of sufficiently high magnitude to provide close to $100 \%$ probability of a tumor. When results were re-examined using the appropriate laboratory-derived reference intervals, diagnostic sensitivity was $97 \%$ (TABLE 1 ).

Any differences in overall diagnostic accuracy of plasma compared to urinary fractionated metanephrines are probably relatively small compared to differences of either test of metanephrines with tests of plasma or urinary catecholamines. Choice of either plasma or urinary fractionated metanephrines may benefit from consideration of additional factors as outlined in TABLE 2.

Dr. Anna Sawka (University of Toronto, Toronto, Canada), who described the experience of investigators at the Mayo Clinic in the diagnosis of pheochromocytoma, contended that while plasma measurements of metanephrines offered high diagnostic sensitivity, the test was not widely available and has lower specificity compared with some other tests, which has a negative impact on cost-effectiveness. ${ }^{18}$ Dr. Karel Pacak (NIH, Bethesda, MD) countered that cost-benefit projections should take into account the potential costs of missing the diagnosis due to use of tests with inadequate sensitivity. 
It was further indicated by Dr. Graeme Eisenhofer (NIH, Bethesda, MD) that because missing a pheochromocytoma can have deadly consequences, one of the most important considerations in the choice of initial test is a high level of reliability that the test will provide a positive result in that rare patient with the tumor. In other words, the initial screening test should be of very high sensitivity. This conversely also provides confidence that a negative result reliably excludes the tumor, thus avoiding the need for multiple or repeat biochemical testing or even costly and unnecessary imaging studies to rule out the tumor, aspects that also should be considered in any cost-benefit analysis. There was general agreement with the conclusions reached by Dr. Sawka in her decision analysis that it was not cost-effective to screen all hypertensives for pheochromocytoma, at least using currently available biochemical tests. In contrast, for individuals genetically predisposed to syndromic pheochromocytoma or identified with an adrenal incidentaloma, the risk or pretest probability of disease is so high that biochemical testing is usually warranted for all such patients.

It was recognized that while measurements of plasma or urinary metanephrines may fail to detect some tumors that synthesize only small amounts of catecholamines or exclusively dopamine, patients with such tumors have an atypical clinical presentation that can benefit from consideration of other testing strategies. As originally established by Dr. Morris Brown (University of Cambridge, Cambridge, UK), present at the breakout session, urinary dopamine is mainly derived from renal extraction of circulating dihydroxyphenylalanine and local conversion to dopamine by aromatic amino-acid decarboxylase. ${ }^{19}$ Thus, measurements of plasma dopamine or its $O$-methylated metabolite, methoxytyramine (which may be measured in urinary and plasma assays of metanephrines), provide better methods for detecting dopamine-producing tumors than measurements of urinary dopamine ${ }^{20}$ In general, though, it should be emphasized that in any test the sensitivity and specificity are inter-related, and dependent on reference intervals. Assessment of diagnostic efficacy is therefore most appropriately achieved by combined examination of sensitivity and specificity in a given clinical context.

A second recommendation arising from the discussions related to the above considerations at the breakout session was that reference intervals for initial tests of urinary or plasma-fractionated metanephrines should be established primarily to ensure optimum diagnostic sensitivity, with specificity being a secondary consideration. Suboptimal diagnostic specificity was nevertheless agreed to present a significant problem. To address the problem of falsepositive results, it was further recommended that testing algorithms should not simply rely on a binary approach for test interpretation (i.e., consideration of whether a test yields a positive or negative result), but should take advantage of the continuous nature of biochemical test results (FIG. 1). For example, while a single elevation of urinary or plasma normetanephrine slightly above the upper reference intervals may only marginally increase the pre- to post-test probability of pheochromocytoma, an elevation of more than fourfold above 
Binary Approach

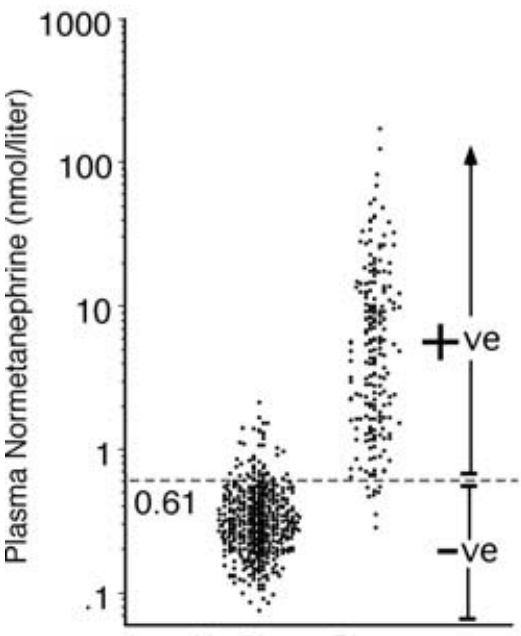

No Pheo Pheo

\section{Continuous Approach}

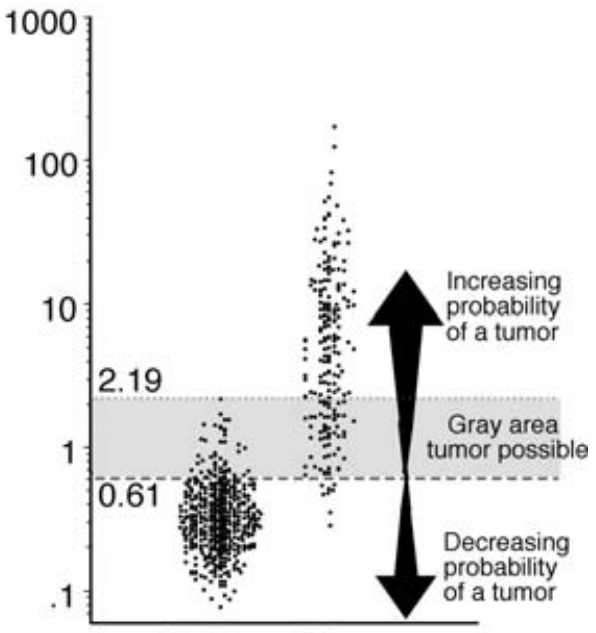

No Pheo Pheo

FIGURE 1. The binary versus continuous approach for the interpretation of biochemical test results is illustrated using measurements of plasma normetanephrine. The horizontal dashed line in both panels illustrates the upper reference limits $(0.61 \mathrm{nmol} / \mathrm{L})$ used to determine whether a plasma concentration of normetanephrine is normal or elevated (negative vs. positive test results according to the binary approach). With some exceptions, noted as follows, pheochromocytoma may be excluded when plasma levels of normetanephrine fall below that level. Exceptions where plasma values in patients with pheochromocytoma fall below the upper reference limit include patients with isolated elevations of plasma metanephrine (metabolite of epinephrine) or methoxytyramine (metabolite of dopamine) or patients with small or microscopic tumors and no other biochemical evidence or signs or symptoms of catecholamine excess. The horizontal dotted line in the panel on the right illustrates the level of plasma normetanephrine $(2.19 \mathrm{nmol} / \mathrm{L})$ above which the test provides $100 \%$ diagnostic specificity. Results that are situated between the two levels (gray area) indicate a tumor is possible, with the probability increasing the higher the value of the test result. Levels above $2.19 \mathrm{nmol} / \mathrm{L}$ provide close to $100 \%$ probability of a tumor.

these intervals is associated with close to $100 \%$ probability of the tumor. In the latter situation more emphasis may be placed on immediately locating the tumor, whereas in the former situation involving test results in the "gray area," there is more of a need to first exclude a false-positive result. Again, some idea of the diagnostic overlap that is seen in weighing sensitivity against specificity is shown by the results in FiguRE 1.

The above recommendations followed lengthy discussion at the breakout session centering on reference intervals, and this was clearly contingent upon the form of sample collection and the appropriate methods for blood sampling. Ms. Debra Harlander (Camden, NY), representing the Pheochromocytoma Support Group, outlined how highly variable instructions for blood sampling from various laboratories and institutions were causing confusion 
among patients and clinicians. At some centers blood sampling was done after 20 min of supine rest using an indwelling i.v. cannula with patients fasting and avoiding acetaminophen (paracetamol). In contrast, elsewhere instructions were inconsistent, with sampling usually carried out with patients seated.

The issue of seated versus supine blood sampling was addressed by Jacques Lenders (St. Radboud University Hospital, Nimegen, the Netherlands), who presented evidence at ISP2005 that blood sampling in the seated position resulted in $25-30 \%$ increases in plasma metanephrines compared to sampling after 20 min of supine rest. It was further shown that use of reference intervals established from blood samples collected in the seated position resulted in reduced diagnostic sensitivity, while testing for pheochromocytoma in seated patients using reference intervals established from samples collected in the supine position substantially reduced diagnostic specificity. It was therefore agreed by all present that reference intervals for plasma free metanephrines should be established using samples collected with the patient in the supine position. To minimize false-positive results, it was further recommended that blood samples for the diagnosis of pheochromocytoma should ideally be collected after $20 \mathrm{~min}$ of supine rest. A caveat to this recommendation was the recognition that blood sampling is usually done by phlebotomists licensed to carry out the procedure according to specific practices. It was therefore proposed that where blood sampling in the seated position returns positive results in the "gray area," repeat testing should be carried out with blood samples collected after $20 \mathrm{~min}$ of supine rest, and where necessary carried out by clinical staff not subject to the same restrictions as phlebotomy technicians.

The use of appropriate normotensive versus hypertensive reference populations, ${ }^{21}$ including (as pointed out by Dr. Sawka) requirements for age adjustments, ${ }^{22}$ and by Dr. Eisenhofer, gender influences,${ }^{23}$ represented other issues touched upon during the breakout session that may assist in interpretation of biochemical tests of catecholamine excess. Taking into account such variables, together with the continuous nature of test results, to calculate changes in preto post-test probabilities of disease for individual patients may be facilitated by advances in test reporting to provide improved approaches to guide clinical decision making for the future. Irrespective of such advances, difficulties in distinguishing true- from false-positive results for tests of catecholamine excess will almost certainly persist, particularly when emphasis is placed on diagnostic sensitivity over specificity.

There was general agreement at the breakout session that imaging studies to search for a suspected tumor should usually not be initiated until biochemical or other evidence of a pheochromocytoma is reasonably compelling. The possibility of false-positive biochemical test results should first be considered and wherever possible excluded. Medications represent common causes of false-positive results, either through direct analytical interference with techniques used to measure catecholamines and catecholamine metabolites, or through pharmacological influences on actual plasma or urinary levels. 
The former causes tend to be method-specific and vary from laboratory to laboratory, making general recommendations on what medications to avoid largely inappropriate. Among the latter causes, tricyclic antidepressants and the $\alpha$-adrenoceptor blocker, phenoxybenzamine, were described in one study as responsible for close to $50 \%$ of all false-positive elevations of plasma and urinary norepinephrine and normetanephrine. ${ }^{24}$ Repeat testing after discontinuation of suspect medications can be used to exclude these as causes of false-positive results. Use of confirmatory follow-up tests, such as urinary fractionated metanephrines to support patterns of increases in initial measurements of plasma metanephrines, or vice versa, were outlined as other approaches to exclude false-positive results, particularly where there is concern about analytical validity.

In all approaches involving multiple tests of catecholamine excess, due consideration should also be given to the inter-dependence of the various analytes that may compromise Bayesian approaches to clinical decision making. A false-positive elevation of urinary normetanephrine due to sympathetic activation is also likely to be associated with false-positive elevations of urinary and plasma norepinephrine and plasma normetanephrine. Thus, while elevations in the latter analytes measured in follow-up tests may serve to confirm the validity of the initial elevated urinary normetanephrine, those additional positive test results do not always provide increased evidence of a pheochromocytoma any more than they provide increased evidence for sympathetic activation. In such situations suppression tests using clonidine or pentolinium can be useful for distinguishing true-positive results due to a pheochromocytoma from falsepositive results due to sympathetic activation. ${ }^{25,26}$ Such tests, although originally developed to distinguish true- from false-positive elevations of plasma catecholamines, may also be applied to distinguish true- from false-positive elevations of urinary metanephrines ${ }^{27}$ or plasma normetanephrine. ${ }^{24}$

\section{TUMOR LOCALIZATION}

Several recent advances in our understanding of pheochromocytoma are now important for physicians to consider when deciding on which imaging modality to use for localization of pheochromocytoma. As outlined at ISP2005 by Dr. Hartmut Neumann (University of Freiburg, Freiburg, Germany) and Dr. Anne-Paule Gimenez-Roqueplo (Université Paris-Descartes, Paris, France), between $12 \%$ and $24 \%$ of patients with apparently sporadic pheochromocytoma may in fact be carriers of germline mutations, indicating hereditary disease with a predisposition for bilateral adrenal, extraadrenal, or multiple tumors. ${ }^{28,29}$ Malignant pheochromocytoma, although rare in multiple endocrine neoplasia type 2 (MEN 2) and von HippelLindau (VHL) syndrome, is common in patients with succinate dehydrogenase B (SDHB) mutations, and is present in about $10 \%$ of patients overall at 
presentation. ${ }^{29-31}$ Further, as outlined at the meeting by Dr. Pierre-François Plouin (Université Paris-Descartes, Paris, France), about 11\% of pheochromocytomas recur as malignant disease. Furthermore, improved biochemical diagnosis of pheochromocytoma requires imaging techniques to locate increasingly smaller tumors (often around $1 \mathrm{~cm}$ in size). In addition, the more frequent use of imaging techniques is leading to increased recognition of "adrenal incidentalomas" as an important clinical finding, requiring follow-up to distinguish functional tumors (e.g., pheochromocytomas) from the more common benign cortical adenomas. ${ }^{32,33}$ Well-defined biochemical phenotypes of adrenal versus extra-adrenal pheochromocytomas and relationships of biochemical findings with tumor mass may further help guide the proper use of anatomical and functional imaging studies. ${ }^{34}$

Below are outlined some of the more important considerations and recommendations on the use of anatomical and functional imaging studies (including their advantages and disadvantages) as discussed during the breakout session. Careful selection of imaging studies should decrease costs and radiation exposure, as well as time and effort required for correct diagnostic localization of tumors.

\section{Anatomical Imaging Studies}

In general, participants felt strongly that localization of pheochromocytoma should only be initiated once the clinical evidence for the tumor is reasonably compelling. For most patients when initial suspicion is based on signs and symptoms, such evidence may include strongly positive biochemical test results (e.g., as illustrated in FIGURE 1) or, when results are not strongly positive, repeated testing after ruling out causes of false-positive results. Imaging studies may be warranted in patients in whom the risk of pheochromocytoma is high because of a hereditary predisposition or a previous history of the tumor, even when biochemical evidence of a pheochromocytoma is less than compelling. In some such patients, imaging studies may be appropriate as part of a periodic surveillance plan, not only for pheochromocytoma, but also to check for other tumors (e.g., kidney tumors in VHL syndrome).

Except for children and pregnant women or rarely in patients with allergies to contrast medium, there was no consensus about whether to use computed tomography (CT) or magnetic resonance imaging (MRI) for the initial localization of a tumor. It was concluded that this depends largely on institutional preference and expertise. Both imaging modalities have excellent sensitivity for the detection of adrenal tumors, but lack adequate specificity for unequivocally confirming a mass as a pheochromocytoma. Neither CT nor MRI can distinguish with clear certainty a functional from a nonfunctional tumor.

Overall, the sensitivity of contrast-enhanced CT in the localization of pheochromocytoma is about $90-95 \%{ }^{35-37}$ Adrenal pheochromocytoma of $1 \mathrm{~cm}$ or larger or metastatic tumors at least $1.0-2.0 \mathrm{~cm}$ in size can be easily 
detected by $\mathrm{CT}$ using narrow collimation. CT densitometry using Hounsfield units (HU) is helpful to distinguish between benign adrenal adenomas and other tumors (often functioning) including metastatic lesions. Thus, a homogeneous mass with a density measurement of less than $10 \mathrm{HU}$ on an unenhanced CT is almost certainly an adenoma. The appearance of homogeneity with soft-tissue density (approximately 40-50 HU) and showing uniform enhancement following intravenous injection of contrast medium is suggestive of a pheochromocytoma, but again it is important to note that it does not distinguish a functional tumor from a nonfunctional one. Larger pheochromocytomas may undergo hemorrhage or necrosis, which results in an inhomogeneous appearance both before and after intravenous injection of contrast medium. Calcification occurs in about $10-15 \%$ of cases.

The advantages of $\mathrm{CT}$ in the localization of pheochromocytoma are the moderate cost and excellent detection of structures surrounding a pheochromocytoma. Data from several different institutions suggest that new nonionic contrast media do not pose a significant risk of a hypertensive crisis. ${ }^{38}$ Consequently, several attendees indicated that they no longer use or recommend $\alpha$-adrenoceptor blockade for such imaging. The recent development of multidetector computed tomography (MDCT) effectively allowing "volume" acquisition of data using 16, 64, or even more detectors has revolutionized CT. Images can now be acquired at great speed, viewed at very fine space intervals, and reformatted to produce multiplanar or $3 \mathrm{D}$ images. This is likely to prove useful, particularly in the identification of small and extra-adrenal tumors.

MRI is also widely used for the diagnosis of adrenal, extra-adrenal, or metastatic pheochromocytoma and has similar sensitivity to CT. ${ }^{37,39}$ The hypervascularity of pheochromocytomas characteristically makes them appear to have a high or intermediate signal intensity on T2-weighted sequences. ${ }^{40} \mathrm{How}-$ ever, it should be noted that such intense signals can be elicited by hemorrhage or hematomas, adenomas, and carcinomas, so an overlap with these tumors and pheochromocytoma must be considered. Moreover, MRI is also a very good imaging modality for the detection of extra-adrenal pheochromocytomas such as intracardiac, juxtacardiac, and juxtavascular pheochromocytoma. This is in part due to the superior contrast resolution of MRI, but also because of the multiplanar acquisition of data without the need for reformatting. MRI can be carried out with or without using intravenous contrast agents, and without requiring $\alpha$-adrenergic blockade. MRI is the preferred initial imaging procedure for pheochromocytoma in children, during pregnancy, or in patients with known allergy to contrast agents.

\section{Functional Imaging Studies}

There is debate about whether functional imaging is necessary once a tumor is located by CT or MRI. The functional imaging test of choice is currently $\left[{ }^{123} \mathrm{I}\right]$-metaiodobenzylguanidine (MIBG) scintigraphy, ${ }^{41,42}$ but as 
outlined above, several considerations may have an impact on the use of such tests. Participants agreed that additional functional imaging studies should be performed in most cases of biochemically proven pheochromocytoma for two main reasons: (1) to prove that the tumor is indeed a pheochromocytoma; and (2) to correctly detect any extension of disease that is not identified by anatomic imaging (e.g., presence of multifocal or bilateral disease or metastatic lesions), which may guide an appropriate therapeutic plan. It should be noted that this recommendation was based on expert opinion, as data for or against the use of routine functional imaging are limited. As reviewed by Dr. James Sisson (University of Michigan, Ann Arbor, MI), a unilateral lesion shown on CT or MRI in the absence of a family history or any evidence of malignancy usually does not reveal additional disease on $\left[{ }^{123} \mathrm{I}\right]$-MIBG scintigraphy ${ }^{43}$ Nevertheless, exceptions do occur and $\left[{ }^{123} \mathrm{I}\right]-\mathrm{MIBG}$ scintigraphy may be specially useful when the biochemical evidence for a pheochromocytoma is unequivocal, but CT or MRI fails to identify the source.

Decisions for imaging should also take into account prior knowledge of the patient. For example, in some patients at risk for extra-adrenal tumors due to germline mutations of SDHB and succinate dehydrogenase D (SDHD) genes or a previous history of paraganglioma, it may be appropriate to include $\left[{ }^{123} \mathrm{I}\right]$-MIBG scintigraphy with CT or MRI as part of the initial imaging evaluation. As indicated by Dr. Karel Pacak, exceptions where [ $\left.{ }^{123} \mathrm{I}\right]-\mathrm{MIBG}$ scintigraphy may not be necessary include solitary adrenal masses of less than $5 \mathrm{~cm}$ associated with elevations of plasma or urinary epinephrine or its metabolite, metanephrine. This suggestion was based on the perception that malignant disease mainly occurs in association with large norepinephrinepredominant tumors, as well as findings that practically all epinephrineproducing pheochromocytomas - including those in patients with MEN 2 - are found in the adrenal gland or are recurrences of previously resected adrenal tumors. $^{34}$

Although no firm recommendations were directed to the use of other functional imaging modalities, these should nevertheless be mentioned because they hold considerable promise, and in certain situations they have already been demonstrated to be of value in the localization of certain cases of the tumor. Such functional positron emission tomography (PET) imaging approaches include use of several imaging agents: 6 - $\left[{ }^{18} \mathrm{~F}\right]$-fluorodopamine, $\left[{ }^{18} \mathrm{~F}\right]-$ dihydroxyphenylalanine, $\left[{ }^{11} \mathrm{C}\right]$-hydroxyephedrine, and $\left[{ }^{11} \mathrm{C}\right]$ epinephrine. ${ }^{44-48}$ All these imaging modalities are highly specific for pheochromocytoma because they depend on uptake into tumor cells by norepinephrine transporters or other transport and storage mechanisms uniquely expressed in chromaffin tissues, including pheochromocytoma tumor cells. Thus, positive results obtained by these methods as well as [ $\left.{ }^{123} \mathrm{I}\right]-\mathrm{MIBG}$ scintigraphy detect pheochromocytoma with almost $100 \%$ specificity.

$\left[{ }^{18} \mathrm{~F}\right]$-fluorodeoxyglucose $\left(\left[{ }^{18} \mathrm{~F}\right]-\mathrm{FDG}\right)$ PET scanning is another approach used to localize pheochromocytoma. $\left[{ }^{18} \mathrm{~F}\right]-\mathrm{FDG}$, however, is a nonspecific 
imaging agent with localization dependent on the higher metabolic rate of tumors compared to surrounding normal tissue. Therefore, the use of this imaging modality should be reserved for those patients in whom either pheochromocytoma cannot be localized by other imaging methods or in patients with rapidly growing tumors that have a high metabolic rate, which often results in excellent detection of those tumors. A disadvantage of PET is that most of the radiopharmaceuticals (except FDG) are not widely available and patients must be referred to a specialized medical center.

Another promising method to localize pheochromocytomas is somatostatin receptor scintigraphy ("OctreoScan"). ${ }^{49,50}$ Although the method appears to have limited use for the localization of solitary pheochromocytomas, it can be useful in patients with metastatic disease or extra-adrenal paragangliomas.

Finally, some of those present at the breakout session indicated, in selected instances, that venous catheterization and sampling studies may add information not readily obtainable by other techniques. ${ }^{51}$ This would include sampling from the adrenal veins in patients with elevated catecholamine or catecholamine metabolites and in whom imaging studies have been uninformative, particularly those with a background of hereditary disease. Reversal of the normally high epinephrine-to-norepinephrine ratio in adrenal venous blood can be particularly useful in such cases for pointing to an intra-adrenal tumor. ${ }^{51}$ Measurements of metanephrines in blood obtained during venous catheterization can provide further advantages over measurements of the parent catecholamines. ${ }^{52}$

In summary, in most patients localization and confirmation of pheochromocytoma should be attempted using at least two imaging modalities. It was generally agreed that anatomical imaging studies (CT or MRI) should be combined with functional (nuclear medicine) imaging studies for optimal localization of primary, recurrent, multiple, or metastatic pheochromocytomas. CT or MRI of the abdomen and pelvis should usually be used first and if they are found to be negative, then CT or MRI of chest and neck and functional imaging should follow. The functional imaging test of choice is $\left[{ }^{123} \mathrm{I}\right]-\mathrm{MIBG}$ scintigraphy, which may be further used to confirm a tumor as a pheochromocytoma and to check for additional multifocal or malignant disease. If MIBG scintigraphy is negative, the diagnosis should be reconsidered, particularly in the light of drug-related false-positive results or the sleep apnea syndrome. Further localization studies should be carried out in major centers and can include PET with specific radiopharmaceuticals, octreotide scintigraphy, or selective venous sampling. If suspicion of a poorly differentiated pheochromocytoma remains, imaging with $\left[{ }^{18} \mathrm{~F}\right] \mathrm{FDG}$ PET may be appropriate.

\section{ACKNOWLEDGMENTS}

The input of all participants in the breakout and final general discussion sessions at ISP2005 is gratefully appreciated. Particular thanks are extended 
to Drs. Stephen Ball, Morris Brown, and Emmanuel Bravo for their valuable suggestions during the preparation of this manuscript. This work was supported in part by the Intramural Research Program of the NIH, National Institute of Neurological Disorders and Stroke, and National Institute of Child Health and Human Development.

\section{REFERENCES}

1. Platts, J.K., P.J. Drew \& J.N. HaRvey. 1995. Death from phaeochromocytoma: lessons from a post-mortem survey. J. Roy. Coll. Physicians Lond. 29: 299-306.

2. Lo, C.Y. et al. 2000. Adrenal pheochromocytoma remains a frequently overlooked diagnosis. Am. J. Surg. 179: 212-215.

3. McNeIL, A.R. et al. 2000. Phaeochromocytomas discovered during coronial autopsies in Sydney, Melbourne and Auckland. Aust. N. Z. J. Med. 30: 648-652.

4. Kaltsas, G.A., D. Papadogias \& A.B. Grossman. 2004. The clinical presentation (symptoms and signs) of sporadic and familial chromaffin cell tumours (phaeochromocytomas and paragangliomas). Front. Horm. Res. 31: 61-75.

5. SJoerdSMA, A. et al. 1959. Studies on the biogenesis and metabolism of norepinephrine in patients with pheochromocytoma. J. Clin. Invest. 38: 31-38.

6. Crout, J.R. \& A. SJoerdSMA. 1964. Turnover and metabolism of catecholamines in patients with pheochromocytoma. J. Clin. Invest. 43: 94-102.

7. EISENHOFER, G. et al. 2003. Pheochromocytoma: rediscovery as a catecholaminemetabolizing tumor. Endocr. Pathol. 14: 193-212.

8. EISENHOFER, G. et al. 1999. Plasma normetanephrine and metanephrine for detecting pheochromocytoma in von Hippel-Lindau disease and multiple endocrine neoplasia type 2. N. Engl. J. Med. 340: 1872-1879.

9. RABER, W. et al. 2000. Diagnostic efficacy of unconjugated plasma metanephrines for the detection of pheochromocytoma. Arch. Intern. Med. 160: 2957-2963.

10. LENDERs, J.W. et al. 2002. Biochemical diagnosis of pheochromocytoma: which test is best? JAMA 287: 1427-1434.

11. SAWKA, A.M. et al. 2003. A comparison of biochemical tests for pheochromocytoma: measurement of fractionated plasma metanephrines compared with the combination of 24-hour urinary metanephrines and catecholamines. J. Clin. Endocrinol. Metab. 88: 553-558.

12. SAWKA, A.M. et al. 2004. A systematic review of the literature examining the diagnostic efficacy of measurement of fractionated plasma free metanephrines in the biochemical diagnosis of pheochromocytoma. BMC Endocr. Disord. 4: 2 .

13. UNGER, N. et al. 2006. Diagnostic value of various biochemical parameters for the diagnosis of pheochromocytoma in patients with adrenal mass. Eur. J. Endocrinol. 154: 409-417.

14. GARDET, V. et al. 2001. Lessons from an unpleasant surprise: a biochemical strategy for the diagnosis of pheochromocytoma. J. Hypertens. 19: 1029-1035.

15. GeRLo, E.A. \& C. SEVENS. 1994. Urinary and plasma catecholamines and urinary catecholamine metabolites in pheochromocytoma: diagnostic value in 19 cases. Clin. Chem. 40: 250-256.

16. DAVIDSON, D.F. 2002. Phaeochromocytoma with normal urinary catecholamines: the potential value of urinary free metadrenalines. Ann. Clin. Biochem. 39: 557566. 
17. GULLER, U. et al. 2006. Detecting pheochromocytoma: defining the most sensitive test. Ann. Surg. 243: 102-107.

18. SAWKA, A.M. et al. 2004. The economic implications of three biochemical screening algorithms for pheochromocytoma. J. Clin. Endocrinol. Metab. 89: 28592866.

19. Brown, M.J. \& D.J. Allison. 1981. Renal conversion of plasma DOPA to urine dopamine. Br. J. Clin. Pharmacol. 12: 251-253.

20. EISENHOFER, G. et al. 2005. Biochemical and clinical manifestations of dopamineproducing paragangliomas: utility of plasma methoxytyramine. J. Clin. Endocrinol. Metab. 90: 2068-2075.

21. Ross, G.A. et al. 1993. Plasma and 24 h-urinary catecholamine concentrations in normal and patient populations. Ann. Clin. Biochem. 30 (Pt. 1): 38-44.

22. SAWKA, A.M. et al. 2005. Measurement of fractionated plasma metanephrines for exclusion of pheochromocytoma: can specificity be improved by adjustment for age? BMC Endocr. Disord. 5: 1.

23. EISENHOFER, G. et al. 1995. Plasma metadrenalines: do they provide useful information about sympatho-adrenal function and catecholamine metabolism? Clin. Sci. (Lond.) 88: 533-542.

24. EISENHOFER, G. et al. 2003. Biochemical diagnosis of pheochromocytoma: how to distinguish true- from false-positive test results. J. Clin. Endocrinol. Metab. 88: 2656-2666.

25. Brown, M.J. et al. 1981. Increased sensitivity and accuracy of phaeochromocytoma diagnosis achieved by use of plasma-adrenaline estimations and a pentolinium-suppression test. Lancet 1: 174-177.

26. BRAVO, E.L. et al. 1981. Clonidine-suppression test: a useful aid in the diagnosis of pheochromocytoma. N. Engl. J. Med. 305: 623-626.

27. Mulinari, R.A. et al. 1987. The clonidine test for the diagnosis of pheochromocytoma: the usefulness of urinary metanephrine measurements. Braz. J. Med. Biol. Res. 20: 43-46.

28. Neumann, H.P. et al. 2002. Germ-line mutations in nonsyndromic pheochromocytoma. N. Engl. J. Med. 346: 1459-1466.

29. AMAR, L. et al. 2005. Genetic testing in pheochromocytoma or functional paraganglioma. J. Clin. Oncol. 23: 8812-8818.

30. Remine, W. et al. 1974. Current management of pheochromocytoma. Ann. Surg. 179: $740-748$.

31. Troncone, L. et al. 1990. The diagnostic and therapeutic utility of radioiodinated metaiodobenzylguanidine (MIBG): 5 years of experience. Eur. J. Nucl. Med. 16: $325-335$.

32. Peppercorn, P.D., A.B. Grossman \& R.H. RezneK. 1998. Imaging of incidentally discovered adrenal masses. Clin. Endocrinol. (Oxford) 48: 379388.

33. Grumbach, M.M. et al. 2003. Management of the clinically inapparent adrenal mass ("incidentaloma"). Ann. Intern. Med. 138: 424-429.

34. EISENhofer, G. et al. 2005. Pheochromocytoma catecholamine phenotypes and prediction of tumor size and location by use of plasma free metanephrines. Clin. Chem. 51: 735-744.

35. Francis, I. \& M. Korobkin. 1996. Pheochromocytoma. Adv. Uroradiol. 34: 11011112.

36. GoldSTEIN, R.E. et al. 1999. Clinical experience over 48 years with pheochromocytoma. Ann. Surg. 229: 755-764; discussion 764-766. 
37. SAHDEV, A. et al. 2005. CT and MR imaging of unusual locations of extra-adrenal paragangliomas (pheochromocytomas). Eur. Radiol. 15: 85-92.

38. MukHERJEe, J.J. et al. 1997. Pheochromocytoma: effect of nonionic contrast medium in CT on circulating catecholamine levels. Radiology 202: 227-231.

39. QUINT, L.E. et al. 1987. Pheochromocytoma and paraganglioma: comparison of MR imaging with CT and I-131 MIBG scintigraphy. Radiology 165: 89-93.

40. VARGHESE, J.C. et al. 1997. MR differentiation of phaeochromocytoma from other adrenal lesions based on qualitative analysis of T2 relaxation times. Clin. Radiol. 52: 603-606.

41. FurutA, N. et al. 1999. Diagnosis of pheochromocytoma using [123I]-compared with [131I]-metaiodobenzylguanidine scintigraphy. Int. J. Urol. 6: 119-124.

42. Ilias, I., B. SHULKIN \& K. PACAK. 2005. New functional imaging modalities for chromaffin tumors, neuroblastomas and ganglioneuromas. Trends Endocrinol. Metab. 16: 66-72.

43. MisKULIN, J. et al. 2003. Is preoperative iodine 123 meta-iodobenzylguanidine scintigraphy routinely necessary before initial adrenalectomy for pheochromocytoma? Surgery 134: 918-922; discussion 922-923.

44. Hoegerle, S. et al. 2002. Pheochromocytomas: detection with 18F DOPA whole body PET: initial results. Radiology. 222: 507-512.

45. PACAK, K. et al. 2001. 6-[18F]fluorodopamine positron emission tomographic (PET) scanning for diagnostic localization of pheochromocytoma. Hypertension 38: $6-8$.

46. Shulkin, B.L. et al. 1999. Pheochromocytomas: imaging with 2-[fluorine18]fluoro-2-deoxy-D-glucose PET. Nucl. Med. 212: 35-41.

47. ManN, G.N. et al. 2006. [(11)C]metahydroxyephedrine and [(18)F]Fluorodeoxyglucose positron emission tomography improve clinical decision making in suspected pheochromocytoma. Ann. Surg. Oncol. 13: 187-197.

48. Trampal, C. et al. 2004. Pheochromocytomas: detection with 11C hydroxyephedrine PET. Radiology 230: 423-428.

49. Mediavilla Garcia, J.D. et al. 1999. A comparative study of 9 cases of adrenal pheochromocytoma and 11 cases of extra-adrenal pheochromocytoma. Rev. Clin. Esp. 199: 343-348.

50. Kaltsas, G. et al. 2001. Comparison of somatostatin analog and metaiodobenzylguanidine radionuclides in the diagnosis and localization of advanced neuroendocrine tumors. J. Clin. Endocrinol. Metab. 86: 895-902.

51. CHEw, S.L. et al. 1994. Bilateral phaeochromocytomas in von Hippel-Lindau disease: diagnosis by adrenal vein sampling and catecholamine assay. Q. J. Med. 87: 49-54.

52. PACAK, K. et al. 2001. A "pheo" lurks: novel approaches for locating occult pheochromocytoma. J. Clin. Endocrinol. Metab. 86: 3641-3646. 